\title{
DYNAMICS OF A TWO SEX POPULATION WITH GESTATION PERIOD
}

Abstract. We investigate a mathematical model of population dynamics for a population of two sexes (male and female) in which new individuals are conceived in a process of mating between individuals of opposed sexes and their appearance is postponed by a period of gestation. The model is a system of two partial differential equations with delay which are additionally coupled by mathematically complicated boundary conditions. We show that this model has a global solution. We also analyze stationary ("permanent") solutions and show that such solutions exist if the model parameters satisfy two nonlinear relations.

1. Introduction. The purpose of the paper is the development and analysis of a mathematical model of a population in which individuals are characterized by sex (male or female) and age. In this model we assume that new individuals are conceived in a process of mating between male and female partners. We also take into account the period of gestation. To simplify the model we are not considering the formation of pairs, so a male individual is allowed to mate any nonpregnant female. Considering the formation of pairs would imply a more sophisticated model (cf. [2]), which is postponed to future research. As we shall see later the model consists essentially of a system of two nonlinear partial differential equations which are nonlinearly coupled by boundary conditions. The presence of a gestation period introduces a delay time. The model has been developed by L. Teglielli [3] and is a generalization of a simpler model proposed by G. Busoni and S. Matucci [1].

1991 Mathematics Subject Classification: 92A15, 35R10, 34K10.

Key words and phrases: population dynamics, mathematical modelling, differential equations with delay, stationary solutions. 
We begin the description of the model by introducing the numerical densities of individuals of each sex as functions of their age, the age of both their parents and time. Let $s_{\mathrm{m}}(a, u, v, t)$ be the distribution at time $t$ of males of age $a$, born from a father whose present age would be $u$ and a mother whose present age would be $v$ (we are not taking into account whether the father or mother are still alive or not, hence $u$ and $v$ refer to virtual age, measuring only time from the birth of these individuals). Analogously $s_{\mathrm{f}}(b, u, v, t)$ is the distribution at time $t$ of females of age $b$, born from a father whose present age would be $u$ and a mother whose present age would be $v$.

From these definitions it is clear that $u>a, v>a, u>b$ and $v>b$. Obviously the age of any individual is bounded: $a \leq a_{l}<\infty, b \leq b_{l}<\infty$. The virtual age of parents $(u$ and $v$ ) has a different upper bound as it measures the time from the moment of birth and not the real age $\left(u \leq 2 a_{l}\right.$ and $v \leq 2 b_{l}$ ).

We denote by $\mu_{\mathrm{m}}(a)$ and $\mu_{\mathrm{f}}(b)$ the mortalities of males and females, respectively. We assume that these functions are also defined for negative $a$ and $b$ to account for mortality of foetuses. This means that $\mu_{\mathrm{m}}=0$ for $a<-g$ and $\mu_{\mathrm{f}}=0$ for $b<-g$, where $g$ is the gestation time.

We now define the total number of males and females of a given age:

$$
\begin{aligned}
M(a, t) & =\int s_{\mathrm{m}}(a, u, v, t) d u d v, \\
F(b, t) & =\int s_{\mathrm{f}}(b, u, v, t) d u d v .
\end{aligned}
$$

To model the mating process, we have to distinguish between pregnant and nonpregnant females. Let $H(b, t)$ be the distribution of pregnant females and $N(b, t)$ the distribution of nonpregnant ones $(H(b, t)+N(b, t)=F(b, t))$. Following Hadeler [2] we assume that the density of mating (density of new pregnancies) is given by the expression

$$
\phi(a, b, t)=2 k(a, b) \frac{M(a, t) N(b, t)}{\int_{0}^{a_{l}} M(u, t) d u+\int_{0}^{b_{l}} N(v, t) d v},
$$

where $k(a, b)$ is the mating factor. Clearly $\phi=0$ if $a$ or $b$ are negative. This gives the following expression for the distribution of pregnant females:

$$
H(b, t)=\int_{t-g}^{t} \int_{0}^{a_{l}} \phi(u, b-(t-s), s) \exp \left(-\int_{s}^{t} \mu_{\mathrm{f}}(b-t+\tau) d \tau\right) d u d s .
$$

For the distribution functions $s_{\mathrm{m}}$ and $s_{\mathrm{f}}$ we have the usual evolution equations

$$
\begin{gathered}
\left(\frac{\partial}{\partial t}+\frac{\partial}{\partial a}+\frac{\partial}{\partial u}+\frac{\partial}{\partial v}\right) s_{\mathrm{m}}(a, u, v, t)=-\mu_{\mathrm{m}}(a) s_{\mathrm{m}}(a, u, v, t) \\
\left(\frac{\partial}{\partial t}+\frac{\partial}{\partial b}+\frac{\partial}{\partial u}+\frac{\partial}{\partial v}\right) s_{\mathrm{f}}(b, u, v, t)=-\mu_{\mathrm{f}}(b) s_{\mathrm{f}}(b, u, v, t)
\end{gathered}
$$


We now present the model of boundary conditions. The functions $s_{\mathrm{m}}(0, u, v, t)$ and $s_{\mathrm{f}}(0, u, v, t)$ are the numbers of newborn males and females, respectively, which were conceived at time $t-g$ by fathers of age $u-g$ and mothers of age $v-g$ and survived the gestation period $[t-g, t]$. The evolution of foetuses is described by the following equation, which takes into account the mortality of foetuses and the mortality of pregnant females:

$$
\begin{aligned}
& \frac{d}{d \bar{t}} s_{j}(\bar{t}-t, u-t+\bar{t}, v-t+\bar{t}, \bar{t}) \\
& \quad=-\left(\mu_{j}(\bar{t}-t)+\mu_{\mathrm{f}}(v-t+\bar{t})\right) s_{j}(\bar{t}-t, u-t+\bar{t}, v-t+\bar{t}, \bar{t}),
\end{aligned}
$$

where $j=\mathrm{m}$ or $\mathrm{f}$. The above equation is supplemented by the initial condition describing the distribution of conceived foetuses at time $t-g$ :

$$
s_{j}(-g, u-g, v-g, t-g)=\beta_{j}(u-g, v-g) \phi(u-g, v-g, t-g),
$$

where $\bar{t} \in[t-g, t] . \beta_{\mathrm{m}}(a, b)$ and $\beta_{\mathrm{f}}(a, b)$ denote the number of individuals of male and female sex, respectively, conceived in the mating of a male of age $a$ and a female of age $b$. As previously we assume that $\beta_{\mathrm{m}}=0$ and $\beta_{\mathrm{f}}=0$ if $a$ or $b$ is negative.

Solving equation (1.5) we obtain

$$
\begin{aligned}
s_{j}(\bar{t}-t, u-t+\bar{t}, v & -t+\bar{t}, \bar{t}) \\
= & \beta_{j}(u-g, v-g) \phi(u-g, v-g, t-g) \\
& \times \exp \left(-\int_{t-g}^{\bar{t}}\left(\mu_{j}(s-t)+\mu_{\mathrm{f}}(v-t+s)\right) d s\right) .
\end{aligned}
$$

This gives the boundary conditions for equations (1.4) at $\bar{t}=t$ :

$$
\begin{aligned}
s_{j}(0, u, v, t)= & \beta_{j}(u-g, v-g) \phi(u-g, v-g, t-g) \\
& \times \exp \left(-\int_{t-g}^{t}\left(\mu_{j}(s-t)+\mu_{\mathrm{f}}(v-t+s)\right) d s\right) .
\end{aligned}
$$

The model presented above was first analyzed by Busoni and Matucci [1]. In that paper only partial analytical and numerical results for a simplified version of the model described by ordinary differential equations with constant coefficients were obtained. Here we would like to extend the previous results to the full model of partial differential equations with variable coefficients.

To obtain a closed model we have to find equations describing the evolution of the densities $M(a, t)$ and $N(b, t)$. To this end, we integrate equations 
(1.4) with respect to $u$ and $v$ to get

$$
\begin{gathered}
\left(\frac{\partial}{\partial t}+\frac{\partial}{\partial a}\right) M(a, t)=-\mu_{\mathrm{m}}(a) M(a, t), \\
\left(\frac{\partial}{\partial t}+\frac{\partial}{\partial b}\right) F(b, t)=-\mu_{\mathrm{f}}(b) F(b, t) .
\end{gathered}
$$

Differentiating equation (1.3) we obtain

$$
\begin{aligned}
&\left(\frac{\partial}{\partial t}+\frac{\partial}{\partial b}\right) H(b, t)=-\mu_{\mathrm{f}}(b) H(b, t)+\int_{0}^{a_{l}} \phi(u, b, t) d u \\
&-\exp \left(-\int_{t-g}^{t} \mu_{\mathrm{f}}(b-t+\tau) d \tau\right) \int_{0}^{a_{l}} \phi(u, b-g, t-g) d u .
\end{aligned}
$$

Since $N=F-H$, we have

$$
\begin{aligned}
&\left(\frac{\partial}{\partial t}+\frac{\partial}{\partial b}\right) N(b, t)=-\mu_{\mathrm{f}}(b) N(b, t)-\int_{0}^{a_{l}} \phi(u, b, t) d u \\
&+\exp \left(-\int_{t-g}^{t} \mu_{\mathrm{f}}(b-t+\tau) d \tau\right) \int_{0}^{a_{l}} \phi(u, b-g, t-g) d u
\end{aligned}
$$

For equations (1.9) and (1.11) we have the following boundary conditions:

$$
\begin{aligned}
& M(0, t)=\int_{g}^{a_{l}} \int_{g}^{b_{l}} s_{\mathrm{m}}(0, u, v, t) d u d v, \\
& N(0, t)=F(0, t)=\int_{g}^{a_{l}} \int_{g}^{b_{l}} s_{\mathrm{f}}(0, u, v, t) d u d v .
\end{aligned}
$$

Because of delay in equation (1.11) the initial distributions have to be given on the whole time interval $[-g, 0]$. Hence we assume that for $t \in[-g, 0]$ we have

$$
M(a, t)=\widehat{M}(a, t), \quad N(b, t)=\widehat{N}(b, t),
$$

where $\widehat{M}$ and $\widehat{N}$ are given functions.

Summarizing, we obtain the following model of population dynamics. There are two species: male individuals with density distribution $M(a, t)$ and nonpregnant females with density $N(b, t)$. These densities satisfy the system of equations

$$
\left(\frac{\partial}{\partial t}+\frac{\partial}{\partial a}\right) M(a, t)=-\mu_{\mathrm{m}}(a) M(a, t),
$$




$$
\begin{aligned}
&\left(\frac{\partial}{\partial t}+\frac{\partial}{\partial b}\right) N(b, t)=-\mu_{\mathrm{f}}(b) N(b, t)-\int_{0}^{a_{l}} \phi(u, b, t) d u \\
&+\exp \left(-\int_{-g}^{0} \mu_{\mathrm{f}}(b+\tau) d \tau\right) \int_{0}^{a_{l}} \phi(u, b-g, t-g) d u .
\end{aligned}
$$

From (1.8) and (1.12) we obtain the boundary conditions at $a=0$ and $b=0$ :

$$
\begin{aligned}
M(0, t+g)= & \int_{0}^{a_{l}-g} \int_{0}^{b_{l}-g} \beta_{\mathrm{m}}(u, v) \phi(u, v, t) \\
& \times \exp \left(-\int_{0}^{g}\left(\mu_{\mathrm{m}}(s-g)+\mu_{\mathrm{f}}(v+s)\right) d s\right) d u d v \\
N(0, t+g)= & \int_{0}^{a_{l}-g} \int_{0}^{b_{l}-g} \beta_{\mathrm{f}}(u, v) \phi(u, v, t) \\
& \times \exp \left(-\int_{0}^{g}\left(\mu_{\mathrm{f}}(s-g)+\mu_{\mathrm{f}}(v+s)\right) d s\right) d u d v .
\end{aligned}
$$

This model is also supplemented by the initial conditions (1.13).

In what follows the biological parameters $\mu_{\mathrm{m}}, \mu_{\mathrm{f}}, \beta_{\mathrm{m}}, \beta_{\mathrm{f}}, k$ and $\phi$ are nonnegative functions of their arguments. The parameters $\beta_{\mathrm{m}}, \beta_{\mathrm{f}}$ and $k$ are bounded functions with the following bounds:

$$
\begin{aligned}
& \sup _{a \in\left(0, a_{l}\right), b \in\left(0, b_{l}\right)} 2 k(a, b) \beta_{\mathrm{m}}(a) \leq K, \\
& \sup _{a \in\left(0, a_{l}\right), b \in\left(0, b_{l}\right)} 2 k(a, b) \beta_{\mathrm{f}}(b) \leq K, \\
& \sup _{a \in\left(0, a_{l}\right), b \in\left(0, b_{l}\right)} 2 k(a, b) \leq K .
\end{aligned}
$$

In addition we assume that

$$
\begin{gathered}
\mu_{\mathrm{m}} \in L_{1}(-g, a) \quad \text { for } a \in\left(-g, a_{l}\right), \quad \lim _{a \rightarrow a_{l}^{-}} \int_{-g}^{a} \mu_{\mathrm{m}}(s) d s=+\infty, \\
\mu_{\mathrm{f}} \in L_{1}(-g, b) \quad \text { for } b \in\left(-g, b_{l}\right), \quad \lim _{b \rightarrow b_{l}^{-}} \int_{-g}^{b} \mu_{\mathrm{f}}(s) d s=+\infty .
\end{gathered}
$$

These assumptions assure that we can consider the evolution problem for $a \in\left(-g, a_{l}\right)$ and $b \in\left(-g, b_{l}\right)$.

2. Existence of solutions. The model described in the previous section will be considered as an evolution problem in the Banach space 
$L^{1}\left(0, a_{l}\right) \times L^{1}\left(0, b_{l}\right)$. Since the equations considered are differential equations with delay it is natural to investigate the whole problem on successive strips (intervals) in time $t \in(i g,(i+1) g]$. Assume that the solution already exists up to time $t=i g$, i.e. we know $M(a, t)$ and $N(b, t)$ for $a \in\left(0, a_{l}\right), b \in\left(0, b_{l}\right)$ and $t \leq i g$. From equations (1.16) we can calculate $M(0, t)$ and $N(0, t)$ for $t \in(i g,(i+1) g]$. Then we can solve equation (1.14) for $t \in(i g,(i+1) g)$ and $a \leq a_{l}$. This solution is given by the expression

$$
M(a, t)=M\left(a_{0}, t_{0}\right) \exp \left(-\sqrt{2} \int_{a_{0}}^{a} \mu_{\mathrm{m}}(s) d s\right),
$$

where

$$
\begin{aligned}
a_{0} & = \begin{cases}0 & \text { for } a \leq t-i g, \\
a-(t-i g) & \text { for } a>t-i g,\end{cases} \\
t_{0} & = \begin{cases}t-a & \text { for } a<t-i g, \\
i g & \text { for } a \geq t-i g .\end{cases}
\end{aligned}
$$

It is seen from the above formulas that in all cases the point $\left(a_{0}, t_{0}\right)$ belongs to the boundary on which the solution is known.

REMARK. From equation (2.1) it is transparent that $M(a, t)=0$ for $a \geq a_{l}$ due to the assumed property of $\mu_{\mathrm{m}}$ (cf. (1.18)).

Now we can proceed to the solution of equation (1.15). Using equation (2.1) we can reduce (1.15) to

$$
\left(\frac{\partial}{\partial t}+\frac{\partial}{\partial b}\right) N(b, t)=-\mu_{\mathrm{f}}(b) N(b, t)-\frac{A N(b, t)}{B+\int_{0}^{b_{l}} N(u, t) d u}+S(b, t),
$$

where

$$
\begin{aligned}
& A=A(b, t)=2 \int_{0}^{a_{l}} k(u, b) M(u, t) d u \\
& B=B(t)=\int_{0}^{a_{l}} M(u, t) d u \\
& S=S(b, t)=\frac{2 N(b-g, t-g) \int_{0}^{a_{l}} k(u, b-g) M(u, t-g) d u}{\int_{0}^{a_{l}} M(u, t-g) d u+\int_{0}^{b_{l}} N(u, t-g) d u} \\
& \quad \times \exp \left(-\int_{-g}^{0} \mu_{\mathrm{f}}(b+\tau) d \tau\right)
\end{aligned}
$$

are known functions for $b \in\left(0, b_{l}\right)$ and $t \leq(i+1) g$.

The following facts are straightforward. 
Lemma 1. If $\|M(\cdot, t)\| \leq m$ and $\|N(\cdot, t)\| \leq n$ for $t \leq i g$, then

$$
\begin{aligned}
M(0, t+g) & \leq K \frac{\|M(\cdot, t)\| \cdot\|N(\cdot, t)\|}{\|M(\cdot, t)\|+\|N(\cdot, t)\|} \leq K \min (\|M(\cdot, t)\|,\|N(\cdot, t)\|) \\
& \leq K \min (m, n), \\
N(0, t+g) & \leq K \frac{\|M(\cdot, t)\| \cdot\|N(\cdot, t)\|}{\|M(\cdot, t)\|+\|N(\cdot, t)\|} \leq K \min (\|M(\cdot, t)\|,\|N(\cdot, t)\|) \\
& \leq K \min (m, n),
\end{aligned}
$$

where $K$ is the constant from estimates (1.17) and $\|\cdot\|$ denotes the $L^{1}$ norm.

Lemma 2. If $\|M(\cdot, t)\| \leq m$ for $t \leq i g$, then

$$
\|M(\cdot, t)\| \leq m(1+K g) \quad \text { for } t \in(i g,(i+1) g) .
$$

Proof. From (2.1) we have

$$
\begin{aligned}
\|M(\cdot, t)\| & \leq \int_{0}^{a_{l}} M\left(a_{0}, t_{0}\right) d u \leq \int_{0}^{a_{l}} M(u, i g) d u+\int_{i g}^{t} M(0, s) d s \\
& \leq m+m g K=m(1+K g) .
\end{aligned}
$$

Iterating the estimate of Lemma 2 we obtain

Corollary. We have

$$
\|M(\cdot, t)\| \leq M_{0}(1+K g)^{i} \quad \text { for } t \in((i-1) g, i g],
$$

where $M_{0}=\sup _{t \in[-g, 0]}\|\widehat{M}(\cdot, t)\|$.

Lemma 3. If $\|M(\cdot, t)\| \leq m$ and $\|N(\cdot, t)\| \leq n$ for $t \leq i g$, then the following estimates hold for $t \in(i g,(i+1) g]$ :

$$
\begin{gathered}
\sup _{b \in\left(0, b_{l}\right)}|A(b, t)| \leq K(1+K g) m, \quad|B(t)| \leq(1+K g) m, \\
\|S(\cdot, t)\| \leq K \frac{\|M(\cdot, t)\| \cdot\|N(\cdot, t)\|}{\|M(\cdot, t)\|+\|N(\cdot, t)\|} \leq K \min (\|M(\cdot, t)\|,\|N(\cdot, t)\|) \\
\leq K \min (m, n) .
\end{gathered}
$$

Theorem 1. Let $\|M(\cdot, t)\| \leq m$ and $\|N(\cdot, t)\| \leq n$ for $t \leq$ ig. Equation (1.15) has a unique solution which exists in the whole strip $t \in(i g,(i+1) g]$, $b \in\left[0, b_{l}\right]$. 
Proof. We rewrite (1.15) as the integral equation

$$
\begin{aligned}
N(b, t)= & N\left(b_{0}, t_{0}\right) \exp \left(-\int_{b_{0}}^{b} \mu_{\mathrm{f}}(s) d s\right) \\
& -\int_{i g}^{t} \exp \left(-\int_{b_{*}}^{b} \mu_{\mathrm{f}}(\sigma) d \sigma\right) \frac{A\left(b_{*}, s\right) N\left(b_{*}, s\right)}{B(s)+\int_{0}^{b_{l}} N(u, s) d u} d s \\
& +\int_{i g}^{t} \exp \left(-\int_{b_{*}}^{b} \mu_{\mathrm{f}}(\sigma) d \sigma\right) S\left(b_{*}, s\right) d s,
\end{aligned}
$$

where

$$
b_{*}= \begin{cases}b-(t-s) & \text { for } b>t-s, \\ 0 & \text { for } b<t-s,\end{cases}
$$

and the point $\left(b_{0}, t_{0}\right)$ is defined as follows:

$$
\begin{aligned}
& b_{0}= \begin{cases}b-(t-i g) & \text { for } b \geq t-i g, \\
0 & \text { for } b<t-i g,\end{cases} \\
& t_{0}= \begin{cases}i g & \text { for } b \geq t-i g, \\
t-b & \text { for } b<t-i g\end{cases}
\end{aligned}
$$

Define the operator $T$ by saying that $T N(b, t)$ is the right hand side of (2.3). Using Lemmas 2 and 3 and the estimate

$$
\begin{aligned}
\left\|\frac{A(\cdot, s) N(\cdot, s)}{B(s)+\int_{0}^{b_{l}} N(u, s) d u}\right\| & \leq \sup _{b}|A(b, s)|\left\|\frac{N(\cdot, s)}{B(s)+\int_{0}^{b_{l}} N(u, s) d u}\right\| \\
& \leq \sup _{b}|A(b, s)| \leq K(1+K g) m
\end{aligned}
$$

we obtain

$$
\|T N(\cdot, t)\| \leq n(1+K g)+K(1+K g) m g+K n g .
$$

This shows that $T$ is bounded and maps all bounded functions $N(b, t)$ for $b \in$ $\left[0, b_{l}\right), t \in(i g,(i+1) g]$ into the sphere of radius $n(1+2 K g)+K g(1+K g) m$. We now prove that for $t>i g$ with $t-i g$ sufficiently small the operator $T$ is a contraction. To this end, we consider two functions $N_{1}(b, t)$ and $N_{2}(b, t)$. Using the fact that $\int_{0}^{b_{l}} N(u, t) d u=\|N(\cdot, t)\|$ we can write

$$
\left\|T N_{1}-T N_{2}\right\| \leq \int_{i g}^{t}\left\|\frac{A N_{1}}{B+\left\|N_{1}\right\|}-\frac{A N_{2}}{B+\left\|N_{2}\right\|}\right\| d s .
$$

Let us estimate the norm under the integral sign: 


$$
\begin{aligned}
& \left\|\frac{A N_{1}}{B+\left\|N_{1}\right\|}-\frac{A N_{2}}{B+\left\|N_{2}\right\|}\right\| \\
& =\left\|\frac{A N_{1}}{B+\left\|N_{1}\right\|}-\frac{A N_{2}}{B+\left\|N_{1}\right\|}+\frac{A N_{2}}{B+\left\|N_{1}\right\|}-\frac{A N_{2}}{B+\left\|N_{2}\right\|}\right\| \\
& \leq\left\|\frac{A}{B+\left\|N_{1}\right\|}\left(N_{1}-N_{2}\right)\right\|+\left\|A N_{2}\left(\frac{1}{B+\left\|N_{1}\right\|}-\frac{1}{B+\left\|N_{2}\right\|}\right)\right\| \\
& \leq \frac{K(1+K g) m}{B_{0}}\left\|N_{1}-N_{2}\right\| \\
& +K(1+K g) m\left\|\frac{1}{B+\left\|N_{1}\right\|} \frac{N_{2}}{B+\left\|N_{2}\right\|}\left(\left\|N_{2}\right\|-\left\|N_{1}\right\|\right)\right\| \\
& \leq \frac{K(1+K g) m}{B_{0}}\left\|N_{1}-N_{2}\right\|+\frac{K(1+K g) m}{B_{0}}\left\|N_{1}-N_{2}\right\|,
\end{aligned}
$$

where

$$
B_{0}=\inf _{s \in(i g,(i+1) g)} B(s)
$$

Hence

$$
\left\|T N_{1}-T N_{2}\right\| \leq(t-i g) \frac{2 K(1+K g) m}{B_{0}}\left\|N_{1}-N_{2}\right\|,
$$

which shows that for $t-i g$ sufficiently small the operator $T$ is a contraction. This proves that equation $(2.3)$ has a local solution in $\left(i g, t_{1}\right]$, where $t_{1}$ is the largest value of $t$ for which $T$ is a contraction. Observe, however, that the contraction constant is independent of the norm of the solution (it depends only on the solution of (1.14)). This shows that the solution can be continued with the same time step onto the whole interval $(i g,(i+1) g]$. We should only comment on the positivity of the constant $B_{0}$. This constant is nothing else than the infimum of the norm of $M(a, t)$ in $(i g,(i+1) g]$. From (2.1) it is straightforward that when the initial data $\widehat{M}(a, t)$ are positive for $t=0$ and for some subinterval in $\left(0, a_{l}\right)$, i.e. if $\|\widehat{M}(\cdot, 0)\|>0$, then $\|M(\cdot, t)\|$ is positive and bounded away from zero for any positive, finite time. This observation proves that $B_{0}>0$.

REMARK. Formula (2.1) which gives the solution to equation (1.14) and Theorem 1 which proves the existence of solution to equation (1.15) deal with integral equations. In general, the solutions to equations (2.1) and (2.3) cannot be differentiated with respect to their arguments, which is necessary if we want to prove the existence of solutions to equations (1.14) and (1.15). For this purpose we have to assume in addition that the initial data are absolutely continuous functions of their arguments. Under these assumptions it can be proved that solutions to equations (2.1) and (2.3) are also absolutely continuous functions, hence differentiable almost everywhere. 
3. The existence of "permanent" solutions. In this section we investigate the existence of stationary solutions to our model, i.e. solutions which are constant in time (such solutions are usually called "permanent"). To find stationary solutions we suppress the dependence on time in equations (1.14) and (1.15). Then we obtain the following system:

$$
\begin{aligned}
\frac{d}{d a} m(a) & =-\mu_{\mathrm{m}}(a) m(a) \\
\frac{d}{d b} n(b) & =-\mu_{\mathrm{f}}(b) n(b)-\frac{2 n(b) \int_{0}^{a_{l}} k(u, b) m(u) d u}{\int_{0}^{a_{l}} m(u) d u+\int_{0}^{b_{l}} n(u) d u} \\
& +\frac{2 n(b-g) \exp \left(-\int_{-g}^{0} \mu_{\mathrm{f}}(b+\tau) d \tau\right) \int_{0}^{a_{l}} k(u, b-g) m(u) d u}{\int_{0}^{a_{l}} m(u) d u+\int_{0}^{b_{l}} n(u) d u}
\end{aligned}
$$

The system of equations (3.1)-(3.2) has to be supplemented by initial conditions which are of the form

$$
\begin{aligned}
m(0)= & \int_{0}^{a_{l}-g} \int_{0}^{b_{l}-g} 2 \beta_{\mathrm{m}}(u, v) k(u, v) \frac{m(u) n(v)}{\int_{0}^{a_{l}} m(u) d u+\int_{0}^{b_{l}} n(u) d u} \\
& \times \exp \left(-\int_{0}^{g}\left(\mu_{\mathrm{m}}(s-g)+\mu_{\mathrm{f}}(v+s)\right) d s\right) d u d v, \\
n(0)= & \int_{0}^{a_{l}-g} \int_{0}^{b_{l}-g} 2 \beta_{\mathrm{f}}(u, v) k(u, v) \frac{m(u) n(v)}{\int_{0}^{a_{l}} m(u) d u+\int_{0}^{b_{l}} n(u) d u} \\
& \times \exp \left(-\int_{0}^{g}\left(\mu_{\mathrm{f}}(s-g)+\mu_{\mathrm{f}}(v+s)\right) d s\right) d u d v, \\
n(b)= & 0 \quad \text { for } b \in[-g, 0) .
\end{aligned}
$$

We begin our analysis by solving a simplified version of the above initial value problem. Namely, the initial conditions (3.3)-(3.4) will be replaced by

$$
m(0)=m_{0}, \quad n(0)=n_{0}
$$

(we retain condition (3.5)).

Equation (3.1) can then be solved explicitly to give

$$
m(a)=m_{0} \exp \left(-\int_{0}^{a} \mu_{\mathrm{m}}(s) d s\right) .
$$

To solve (3.2) we proceed as in the time dependent case, i.e. we find a solution on consecutive intervals $(i g,(i+1) g)$. Using (3.7), we can write the 
solution on $(0, g)$ in the form

$$
n(b)=n_{0} \exp \left(-\int_{0}^{b} \mu_{\mathrm{f}}(s) d s-\frac{2 m_{0}}{m_{0} A+X} \int_{0}^{b} A_{1}(s) d s\right),
$$

where

$$
\begin{aligned}
A & =\int_{0}^{a_{l}} \exp \left(-\int_{0}^{u} \mu_{\mathrm{m}}(s) d s\right) d u \\
A_{1}(b) & =\int_{0}^{a_{l}} k(u, b) \exp \left(-\int_{0}^{u} \mu_{\mathrm{m}}(s) d s\right) d u \\
X & =\int_{0}^{b_{l}} n(u) d u .
\end{aligned}
$$

Observe that $A$ is a constant which depends only on the form of the function $\mu_{\mathrm{m}}(s)$. Also $A_{1}(s)$ is a known function. The constant $X$ is of course unknown and depends on the solution. In this sense (3.8) is an integral equation which has to be solved. But since $X$ is a constant we can consider the solution (3.8) as a function of $X$. We shall show further that $n(b)$ can be found as a known function of $X$ on the whole interval $\left(0, b_{l}\right)$. Integrating this function we obtain an algebraic equation in $X$. It can be shown that this algebraic equation has a solution, which gives a value of $X$.

First, however, let us construct $n(b)$ on $(g, 2 g)$. On this interval equation (3.2) has the form

$$
\frac{d}{d b} n(b)=-\mu_{\mathrm{f}}(b) n(b)-\frac{2 m_{0} n(b) A_{1}(b)}{m_{0} A+X}+\frac{2 m_{0} n(b-g) A_{2}(b-g)}{m_{0} A+X},
$$

where

$$
A_{2}(s)=A_{1}(s) \exp \left(-\int_{0}^{g} \mu_{\mathrm{f}}(s+\tau) d \tau\right) .
$$

Solving (3.9) we obtain

$$
\begin{aligned}
n(b)= & n_{0} \exp \left(-\int_{0}^{b} \mu_{\mathrm{f}}(s) d s-\frac{2 m_{0}}{m_{0} A+X} \int_{0}^{b} A_{1}(s) d s\right) \\
& +n_{0} \int_{g}^{b} \exp \left(-\int_{s}^{b} \mu_{\mathrm{f}}(\sigma) d \sigma-\frac{2 m_{0}}{m_{0} A+X} \int_{s}^{b} A_{1}(\sigma) d \sigma\right) \\
& \times \exp \left(-\int_{0}^{s-g} \mu_{\mathrm{f}}(\sigma) d \sigma-\frac{2 m_{0}}{m_{0} A+X} \int_{0}^{s-g} A_{1}(\sigma) d \sigma\right) \\
& \times \frac{2 m_{0}}{m_{0} A+X} A_{2}(s-g) d s .
\end{aligned}
$$


The above expression is a complicated but known function of $X$. It is clear that this procedure can be iterated giving the solution to (3.2) on successive intervals $(i g,(i+1) g)$. Hence the solution to $(3.2)$ can be written on the whole interval $\left(0, b_{l}\right)$ as

$$
n(b)=n_{0} G\left(b, m_{0}, X\right),
$$

where $G\left(b, m_{0}, X\right)$ is a known function of its arguments. Integrating this solution on $\left(0, b_{l}\right)$ we obtain a nonlinear algebraic equation

$$
X=\mathbb{F}(X),
$$

where $\mathbb{F}$ is a known function of $X$. We can now prove that equation (3.12) has a solution. Inspecting formulas (3.8) and (3.10) we find that $\mathbb{F}(X)$ is a continuous function of $X$. We also have $\mathbb{F}(0)>0$. In addition, $\mathbb{F}(X)$ is a bounded function for all nonnegative $X$ (for $X \rightarrow \infty, G\left(b, m_{0}, X\right) \leq 1$ ). Thus $X-\mathbb{F}(X)$ is negative for $X=0$ and positive for sufficiently large $X$. Since $X-\mathbb{F}(X)$ is continuous there exists $X$ such that $X=\mathbb{F}(X)$. This proves that equation (3.2) with conditions (3.5)-(3.6) has a solution.We do not, however, know if this solution is unique.

To solve the original problem (3.1)-(3.5) we have to show that there is a set of initial conditions $\left(m_{0}, n_{0}\right)$ which satisfy (3.3) and (3.4). These equations can be rewritten in the form

$$
\begin{aligned}
m_{0} & =\frac{m_{0} n_{0} Y_{\mathrm{m}}\left(m_{0}, X\right)}{m_{0} A+n_{0} Y\left(m_{0}, X\right)}, \\
n_{0} & =\frac{m_{0} n_{0} Y_{\mathrm{f}}\left(m_{0}, X\right)}{m_{0} A+n_{0} Y\left(m_{0}, X\right)},
\end{aligned}
$$

where

$$
\begin{aligned}
Y\left(m_{0}, X\right) & =\int_{0}^{b_{l}} G\left(b, m_{0}, X\right) d b \\
Y_{\mathrm{m}}\left(m_{0}, X\right) & =\int_{0}^{a_{l}-g} \int_{0}^{b_{l}-g} 2 \beta_{\mathrm{m}}(u, v) k(u, v) G\left(v, m_{0}, X\right) \\
& \times \exp \left(-\int_{0}^{u} \mu_{\mathrm{m}}(s) d s-\int_{0}^{g}\left(\mu_{\mathrm{m}}(s-g)+\mu_{\mathrm{f}}(v+s)\right) d s\right) d u d v \\
Y_{\mathrm{f}}\left(m_{0}, X\right)= & \int_{0}^{a_{l}-g} \int_{0}^{b_{l}-g} 2 \beta_{\mathrm{f}}(u, v) k(u, v) G\left(v, m_{0}, X\right) \\
& \times \exp \left(-\int_{0}^{u} \mu_{\mathrm{m}}(s) d s-\int_{0}^{g}\left(\mu_{\mathrm{f}}(s-g)+\mu_{\mathrm{f}}(v+s)\right) d s\right) d u d v .
\end{aligned}
$$


From (3.13) we obtain

$$
\begin{aligned}
m_{0}\left(m_{0} A+n_{0}\left(Y\left(m_{0}, X\right)-Y_{\mathrm{m}}\left(m_{0}, X\right)\right)\right) & =0, \\
n_{0}\left(m_{0}\left(A-Y_{\mathrm{f}}\left(m_{0}, X\right)\right)+n_{0} Y\left(m_{0}, X\right)\right) & =0 .
\end{aligned}
$$

Because we are interested in nontrivial solutions, we have to solve the system

$$
\begin{aligned}
m_{0} A+n_{0}\left(Y\left(m_{0}, X\right)-Y_{\mathrm{m}}\left(m_{0}, X\right)\right) & =0 \\
m_{0}\left(A-Y_{\mathrm{f}}\left(m_{0}, X\right)\right)+n_{0} Y\left(m_{0}, X\right) & =0 .
\end{aligned}
$$

Since

$$
n_{0}=\frac{X}{Y\left(m_{0}, X\right)}
$$

we can rewrite the above equations in terms of new variables $\left(m_{0}, X\right)$ :

$$
\begin{aligned}
m_{0} A Y\left(m_{0}, X\right)+X\left(Y\left(m_{0}, X\right)-Y_{\mathrm{m}}\left(m_{0}, X\right)\right) & =0, \\
m_{0}\left(A-Y_{\mathrm{f}}\left(m_{0}, X\right)\right)+X & =0 .
\end{aligned}
$$

A nontrivial solution to this system exists only if

$$
A Y_{\mathrm{m}}\left(m_{0}, X\right)+Y_{\mathrm{f}}\left(m_{0}, X\right)\left(Y\left(m_{0}, X\right)-Y_{\mathrm{m}}\left(m_{0}, X\right)\right)=0 .
$$

Because the functions $Y\left(m_{0}, X\right), Y_{\mathrm{m}}\left(m_{0}, X\right)$ and $Y_{\mathrm{f}}\left(m_{0}, X\right)$ are nonnegative, equation (3.15) can be satisfied only if

$$
Y\left(m_{0}, X\right)<Y_{\mathrm{m}}\left(m_{0}, X\right) .
$$

Equation (3.15) in general has a whole family of solutions depending on one parameter. On the other hand if (3.15) holds then system (3.13) also has a one-parameter family of solutions. It is not straightforward that these two families have a nonempty intersection. In addition, to obtain a nonnegative solution to equations (3.13) we have to assume (3.16) and

$$
A<Y_{\mathrm{f}}\left(m_{0}, X\right) .
$$

To summarize, a solution to the stationary problem (3.1)-(3.5) is constructed in the following way:

1. We solve equations (3.1) and (3.2) to find the function $G\left(b, m_{0}, X\right)$. This enables us to find the functions $Y\left(m_{0}, X\right), Y_{\mathrm{m}}\left(m_{0}, X\right)$ and $Y_{\mathrm{f}}\left(m_{0}, X\right)$.

2. We check conditions (3.16) and (3.17). If they hold we solve the nonlinear equation (3.15) and find a one-parameter family of its solutions $X\left(m_{0}\right)$.

3. We reduce system (3.13) to the nonlinear equation

$$
m_{0}\left(A-Y_{\mathrm{f}}\left(m_{0}, X\left(m_{0}\right)\right)\right)+X\left(m_{0}\right)=0 .
$$

Solution of this equation gives the desired initial value $m_{0}$.

4. Knowing $m_{0}$ and $X$ we calculate $n_{0}$ from (3.14). Solutions of equations (3.1) and (3.2) with these initial data solve our original problem. 
Let us observe that the strategy is not effective, for there may be no solution to equation (3.15) or (3.18). It even seems that there is no effective way to find solutions to these equations. Because these solutions depend on the functions $Y, Y_{\mathrm{m}}$ and $Y_{\mathrm{f}}$ which on the other hand depend on the biological parameters $\beta_{\mathrm{m}}, \beta_{\mathrm{f}}, k, \mu_{\mathrm{m}}$ and $\mu_{\mathrm{f}}$ it is clear that the requirement that the solutions exist puts severe restrictions on these parameters which cannot be made more explicit.

Acknowledgments. This work was partially supported by C. N. R.Gruppo Nazionale per la Fisica Matematica. The second author would like to acknowledge C. N. R. for sponsoring his stay in Italy and the hospitality of the University of Florence where this work was completed. The first author's work was also supported by the Ministero per la Università e la Ricerca Scientifica of Italy.

\section{References}

[1] G. Busoni and S. Matucci, Population dynamics with delay, Studi Urbinati I (1997), 119-137 (paper presented at the meeting "La matematica nei problemi dell'ambiente, della biologia e della medicina", Urbino, 1996).

[2] K. P. Hadeler, Pair formation models with maturation period, J. Math. Biology 32 (1993), 1-15.

[3] L. Teglielli, Dinamica di popolazioni a due sessi, Ricerca di soluzioni per sistenti, Università di Firenze, 1998.

Giorgio Busoni

Dipartimento di Matematica "Ulisse Dini"

Università di Firenze

Viale G. B. Morgagni 67/A

50134 Firenze, Italy

E-mail: busoni@udini.math.unifi.it
Andrzej Palczewski

Department of Mathematics

Warsaw University

Banacha 2

02-097 Warszawa, Poland

E-mail: apalczew@mimuw.edu.pl 